\title{
Small Motion Detection and Non-Contact Vital Signs Monitoring with Continuous Wave Doppler Radars
}

\author{
Ibrahim Seflek ${ }^{1, *}$, Yunus Emre Acar ${ }^{2}$, Ercan Yaldiz ${ }^{1}$ \\ ${ }^{I}$ Department of Electrical and Electronics Engineering, Faculty of Engineering and Nature Sciences, \\ Konya Technical University, \\ 42130 Konya, Turkey \\ ${ }^{2}$ Department of Electrical and Electronics Engineering, Faculty of Technology, Selcuk University, \\ 42079 Konya, Turkey \\ iseflek@ktun.edu.tr
}

\begin{abstract}
Radars have become devices that one can come across in any environment at any moment. This means that they enter to all areas of life and even in the field of medicine and will be used more intensively in the future. Especially, the attention has been drawn to that they are suitable for the noncontact vital signs monitoring. In this study, two radar structures operating at $24 \mathrm{GHz}$ (Radar 1) and $2.4 \mathrm{GHz}$ (Radar 2) frequencies are used. Radar 1 structure is created on a printed circuit board (PCB), whereas Radar 2 is obtained by combining discrete components. The $8.5 \mathrm{~mm}$ movement performed with the aid of a test mechanism is detected by two radars with percentage errors (PEs) of $2.58 \%$ and $6.23 \%$, respectively. For the $0.25 \mathrm{~Hz}$ vibration frequency, the error is the same for both radars and is $2.4 \%$. In measurements taken from a healthy human subject, Radar 1 finds a respiration rate with $1.85 \%$ of PE and heart beat rate with $6.17 \%$ of PE. In Radar 2, these values are $2.35 \%$ and $8.24 \%$, respectively. From the measurement results, it is seen that the resolution of Radar 1 is better than that of Radar 2. The results also indicate that small motion detection and vital signs monitoring are carried out successfully.
\end{abstract}

Index Terms-Displacement measurement; Doppler radar; Non-contact measurement; Vital signs monitoring.

\section{INTRODUCTION}

The elderly population is increasing in proportion to the increasing world population. Human life is prolonged thanks to advancing medical technology, and according to United Nations (UN) data, the proportion of people aged 60 and over in the world is expected to reach 16.5 percent in 2030 and 22 percent in 2050 [1]. Health expenditures will increase in parallel with the increasing population of the elderly and monitoring of the vital functions of people will become an important need. Respiratory rate (RR), heart rate (HR), and blood oxygen saturation $\left(\mathrm{SpO}_{2}\right)$ constitute physiological parameters for monitoring human health. In the present case, contact devices, such as pulse oximeters, piezoelectric sensors, electrocardiography (ECG), photoplethysmography (PPG), and respiratory belt, perform vital signs monitoring. This is uncomfortable for the patient. It causes extra injuries, especially in neonatal units and burn

Manuscript received 30 December, 2019; accepted 30 March, 2020. cases.

Radar technology has been used for civilian applications since the mid-1970s [2]. Especially in the last 20 years, it has been developed in terms of both hardware and software and adapted to all areas of life. The automotive sector, industrial applications, and the medical field have also been affected [3]. In particular, radars have been proposed to monitor vital signs for eliminating the disturbance caused by contact sensors. The use of radars in the medical field allows the patient to move freely. It reduces maintenance costs and is particularly convenient for medical professionals.

Doppler radar is the basis of non-contact vital signs measurements. The signal transmitted from the radar reaches the target and is reflected back to the radar in a modulated form. The signal received by the radar contains a lot of information about the target. When the human is selected as the target, the reflected signal due to the small vibrations of the chest wall contain information on respiration and heartbeat, which constitute the vital signals of the human being. Therefore, it can be used in the detection of vital signs.

On this basis, many different studies have been put forward a few different radar types [4]-[7]. Radar noncontact measurements are performed in cases such as sleep apnea problem [8], [9], sudden infant death syndromes [10], elderly and patient home care [11], [12], detection of lives under the earthquake debris [13], through wall live detection [14], [15], and surveillance of the target [16]. The success of the studies is very promising for the future and it has been shown that in the cases the rate of intervention can be increased. Furthermore, it has been shown that the studies on the animal subjects can be accomplished by radar [17].

In this paper, two continuous wave Doppler radars operating at $2.4 \mathrm{GHz}$ and $24 \mathrm{GHz}$ are used. Firstly, small displacement motion due to a mechanism operating at a fixed frequency is detected. Then, vital signs are found out utilizing a human subject. The purpose of using two radars of different frequency is to determine how frequencies of radars are sensitive to small displacements. For a human, the frequency and amplitude of displacement of the body surface due to breath and heartbeat are from $0.1 \mathrm{~Hz}$ to 
$0.5 \mathrm{~Hz}$ and from $5 \mathrm{~mm}$ to $15 \mathrm{~mm}$ due to respiration, whereas these values are between $0.8 \mathrm{~Hz}-2 \mathrm{~Hz}$ and $0.45 \mathrm{~mm}-0.7 \mathrm{~mm}$ for the heartbeat [18].

This paper is organized as follows. Section II describes the principle of continuous wave Doppler radar operation. The experimental setup and the signal processing are presented in Section III. The experimental results obtained are submitted in Section IV. The study is evaluated in Section V.

\section{PRINCIPLES OF CONTINUOUS WAVE DOPPLER RADAR}

Figure 1 shows a block diagram of a basic continuous wave $(\mathrm{CW})$ Doppler radar. A CW Doppler radar transmits a sinusoidal wave to the target. The wave reflected from the target is received by the receiving antenna with a tiny but meaningful time delay depending on the distance. The phase difference between the transmitted and received signal is directly related to the target movement. The signal transmitted from the radar is expressed as follows

$$
S_{T X}(t)=A_{T} \cos \left(2 \pi f_{c} t+\phi(t)\right)
$$

where $A_{T}$ indicates the amplitude of the transmitted signal, $f_{c}$ is the frequency of the signal, and $\phi(t)$ is the initial phase noise. The radar signal reflected from the target is received by the receiver antenna. The received signal is expressed as follows:

$$
\begin{gathered}
S_{R X}(t)= \\
=A_{R} \cos \left(2 \pi f_{c} t-\frac{4 \pi d_{0}}{\lambda}-\frac{4 \pi x(t)}{\lambda}-\phi\left(t-\frac{2 d(t)}{c}\right)\right), \\
d(t)=d_{0}+x(t) .
\end{gathered}
$$

In (2), $A_{R}$ represents the amplitude of the received signal and $d_{0}$ is the nominal distance between the radar and the target. In addition, $\lambda$ is carrier wavelength and $x(t)$ refers to the chest wall movement, including breathing and heart movement. $\phi\left(t-\frac{2 d(t)}{c}\right)$ is the delayed phase noise.

A replica of the signal sent from the transmitter is used for the mixer in the receiver with the received signal. The signal obtained at the mixer output is called the "baseband signal" and is expressed as follows

$$
B(t)=\cos \left(\beta+\frac{4 \pi x(t)}{\lambda}+\Delta \phi(t)\right)
$$

where $\beta=4 \pi d_{0} / \lambda$. This is the sum of the phase shift at the nominal distance $d_{0}$ to the source. $\Delta \phi(t)=\phi(t)-\phi(t-2 d(t) / c)$ is the residual phase noise. The phase noise is a characteristic feature of any signal source. Especially in short range, since the $2 d(t) / c$ is very small, its effect can be neglected. The phase noise from the transmitter and receiver is also small and eliminates each other in the baseband. This is known as the range correlation effect. In order to benefit from this phenomenon, it is necessary to use the same source in the transmitter and receiver.

Equation (4) shows the baseband signal output, the sensitivity of the radar depends on the nominal distance. This results in optimal and null point output of the radar. If $\beta$ is an odd multiple of $\pi / 2$ and $x(t)$ is much smaller than $\lambda$, the optimum output phase demodulation sensitivity is achieved and the baseband output is proportional to the chest wall displacement. However, $\beta$ is an even multiple of $\pi / 2$ and $x(t)$ is much smaller than $\lambda$, the sensitivity is very low. Null points occur and the output is no longer proportional to the displacement that changes over time. In order to eliminate this problem of the radar, a quadrature receiver system has been proposed [19]. Figure 2 represents the block diagram of the quadrature radar structure used in the study.

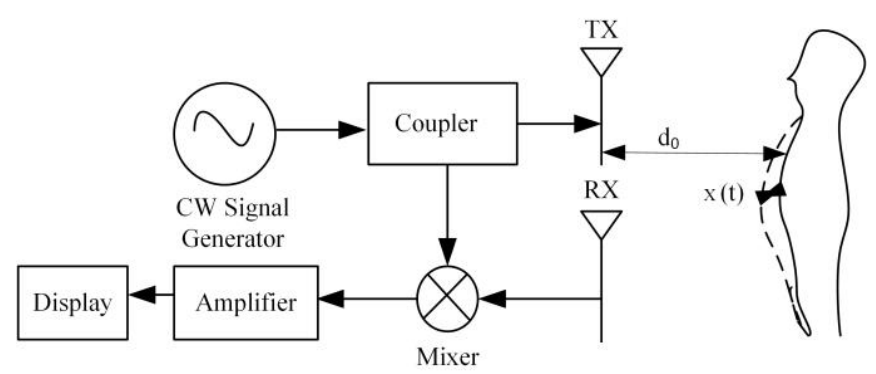

Fig. 1. Block diagram of basic continuous wave Doppler radar.

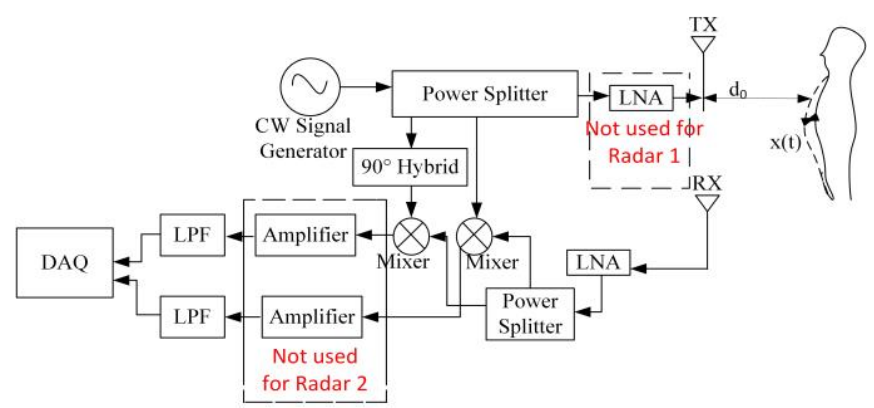

Fig. 2. Block diagram of the quadrature radar structure used in the study.

This system has two outputs that have 90-degree phase difference from each other. These outputs are called "inphase" (I) and "quadrature phase" (Q). The radar baseband outputs are expressed in the following equations:

$$
\left\{\begin{array}{l}
B_{I}(t)=A_{I} \cos \left(\beta+\frac{4 \pi x(t)}{\lambda}+\Delta \phi(t)\right), \\
B_{Q}(t)=A_{Q} \sin \left(\beta+\frac{4 \pi x(t)}{\lambda}+\Delta \phi(t)\right),
\end{array}\right.
$$

where $A_{I}$ and $A_{Q}$ are the signal amplitudes. In (5), one of the outputs always indicates the optimum point. However, if $\beta$ is an integer multiple of $\pi / 4$, it does not have the optimum sensitivity at the two outputs, resulting in misinterpretation of motion. The arctangent demodulation method solves this problem and the phase obtained by combining the two signals is directly proportional to the target movement. The method can be written as follows

$$
\phi(t)=\arctan \left(\frac{B_{Q}(t)}{B_{I}(t)}\right)=\beta+\frac{4 \pi x(t)}{\lambda}+\Delta \phi(t) .
$$

For the method to be successful, the radar must be calibrated appropriately. It is also important to eliminate the 
DC offset generated by the hardware and surrounding objects. I and Q having phase and amplitude matching form an arc or circle depending on the carrier wavelength (frequency). Phase and amplitude imbalances cause the circle to return to the ellipse or even to deteriorate. I/Q plots of simulations for balanced and imbalanced phase and amplitude, respectively, are shown in Fig. 3.

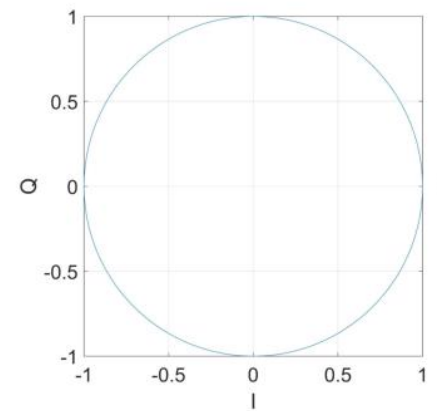

(a)

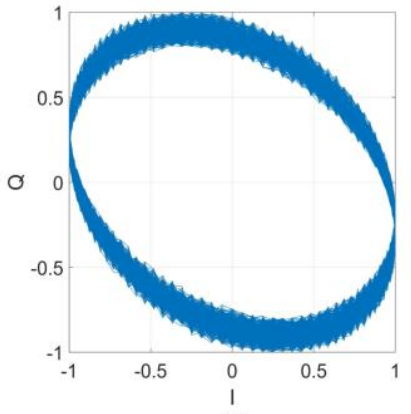

(b)
Fig. 3. (a) I/Q plot with simulated phase and amplitude balanced; (b) I/Q plot with simulated phase and amplitude imbalanced.

The operating frequency of the radar is an important factor for resolution. $4 \pi x(t) / \lambda$ is the amount of phase modulation in radians and $x(t)$ represents the target movement. As shown in (6), higher frequency means lower wavelength and greater phase modulation. Greater phase modulation in the complex I-Q plane creates a longer arc. Figure 4 illustrates this situation.

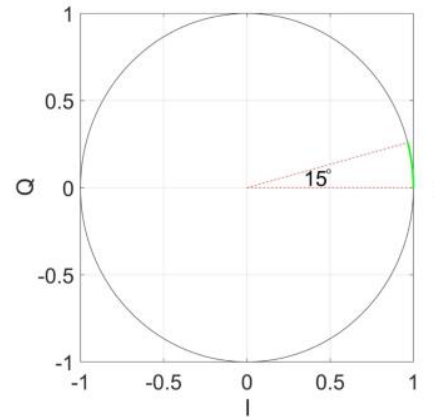

(a)

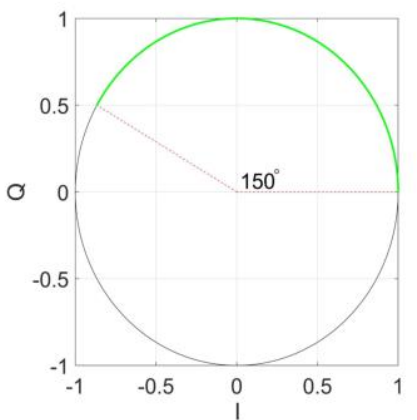

(b)
Fig. 4. (a) Simulated I/Q plot of a motion for $2.4 \mathrm{GHz}$; (b) Simulated I/Q plot of a motion for $24 \mathrm{GHz}$.

The phase changes in the respiration and heart rate calculated for the $2.4 \mathrm{GHz}$ and $24 \mathrm{GHz}$ radars used are shown in Table I.

TABLE I. PHASE CHANGES FOR RESPIRATION AND HEARTBEAT.

\begin{tabular}{|c|c|c|}
\hline Radar & $\begin{array}{c}\text { Phase } \\
(\text { Respiration) }\end{array}$ & Phase (Heartbeat) \\
\hline $2.4 \mathrm{GHz}$ & $28.8^{\circ}-86.4^{\circ}$ & $2.59^{\circ}-4.03^{\circ}$ \\
\hline $24 \mathrm{GHz}$ & $288^{\circ}-864^{\circ}$ & $25.9^{\circ}-40.3^{\circ}$ \\
\hline
\end{tabular}

\section{MeAsurement SetuP AND SignAl Processing}

Two radars operating at two different frequencies are utilized for the study. The first radar utilized for measurements is the RF-Beam K-LC6 radar module [20]. The radar is operated in single tone at $24 \mathrm{GHz}$. Low noise amplifier (LNA) is used in the receiver block to increase the sensitivity of the received signal. In addition, the receiver has a quadrature design architecture. Since the I/Q channel outputs have low amplitudes, baseband amplifier is used for both channels. With the designed $4^{\text {th }}$ order Butterworth lowpass filter, the signal is made ready for the acquisition of vital signs. Identical filters in both channels have a cut-off frequency of $30 \mathrm{~Hz}$ and a gain of 10 . Furthermore, before filtering, both channels were amplified approximately 100 times with the AD620-based commercial voltage gain amplifier. The DC level between the channels is manually corrected with this structure.

The other radar utilized is composed of discrete components with the operating frequency of $2.4 \mathrm{GHz}$. Siglent SSG3032X is preferred as the signal source. The signal generated from the signal source is sent to a splitter. One of the outputs of the splitter is sent to the transmitting antenna, while the other output is sent to a 90-degree hybrid for the quadrature receiver architecture. LNAs have been used to amplify both the transmitter and the signal. Hybrid outputs are used for down-conversion in mixers of the receiver. The received signal is down-converted to baseband with mixers. Using the low-pass filter, the I/Q signals in the desired frequency range are obtained. The radar structures implemented for the measurements are shown in Fig. 5 and Fig. 6.

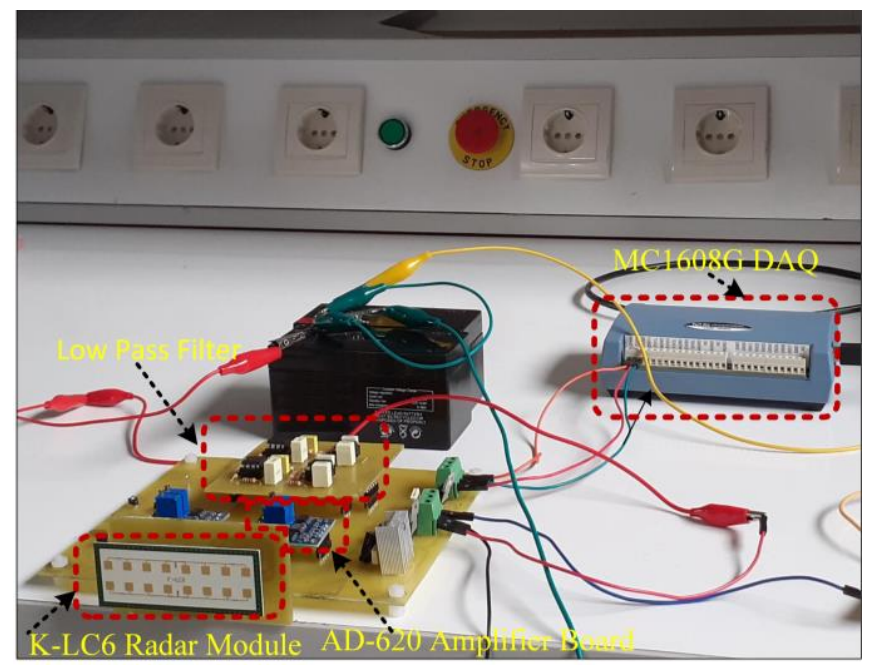

Fig. 5. The implemented $24 \mathrm{GHz}$ radar structure.

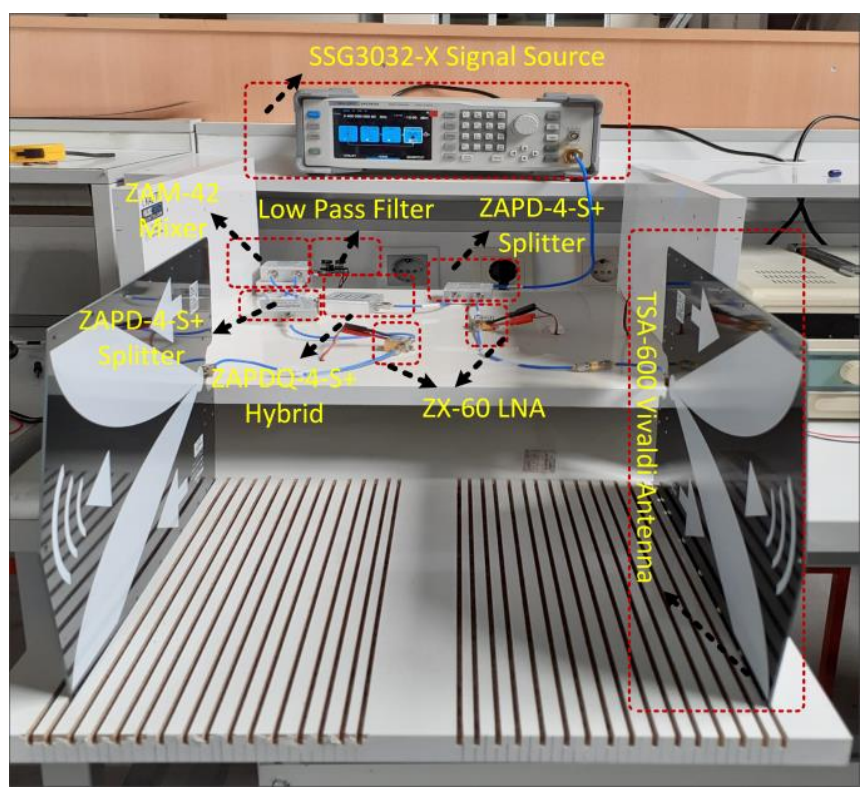

Fig. 6. The implemented $2.4 \mathrm{GHz}$ radar structure. 
Considering the Nyquist criterion to digitize baseband signals, 60 samples/s is sufficient for filtered output from 0 $\mathrm{Hz}$ to $30 \mathrm{~Hz}$. However, 500 samples/s sampling frequency is used for better digitization. Digitization is performed with MC1608G DAQ of Measurement and Computing. The components used for the both radar structures are listed in Table II.

TABLE II. COMPONENT LIST OF RADARS

\begin{tabular}{|c|c|}
\hline Radar 1 (24 GHz) & Radar 2 (2.4 GHz) \\
\hline $\begin{array}{c}\text { RF Beam K-LC6 Radar } \\
\text { Transceiver Module }\end{array}$ & $\begin{array}{c}\text { Siglent SSG3032X Signal } \\
\text { Source }\end{array}$ \\
\hline $\begin{array}{c}\text { AD-620 Based Commercial } \\
\text { Voltage Gain Amplifier }\end{array}$ & ZAPD-4-S+ Splitter \\
\hline $\begin{array}{c}\text { Two Channel Active Filter Board } \\
\text { with 30 Hz Cutoff Frequency }\end{array}$ & ZAPDQ-4-S+ Hybrid \\
\hline MC1608G DAQ & ZAM-42 Mixer \\
\hline- & ZX-60 LNA \\
\hline- & TSA-600 Vivaldi Antenna \\
\hline- & MC1608G DAQ \\
\hline- &
\end{tabular}

The digitized raw data are recorded for signal processing. Although the same principles are used for two different radars, there are differences in algorithms that improve performance. Flow chart of the algorithms is given Fig. 7.

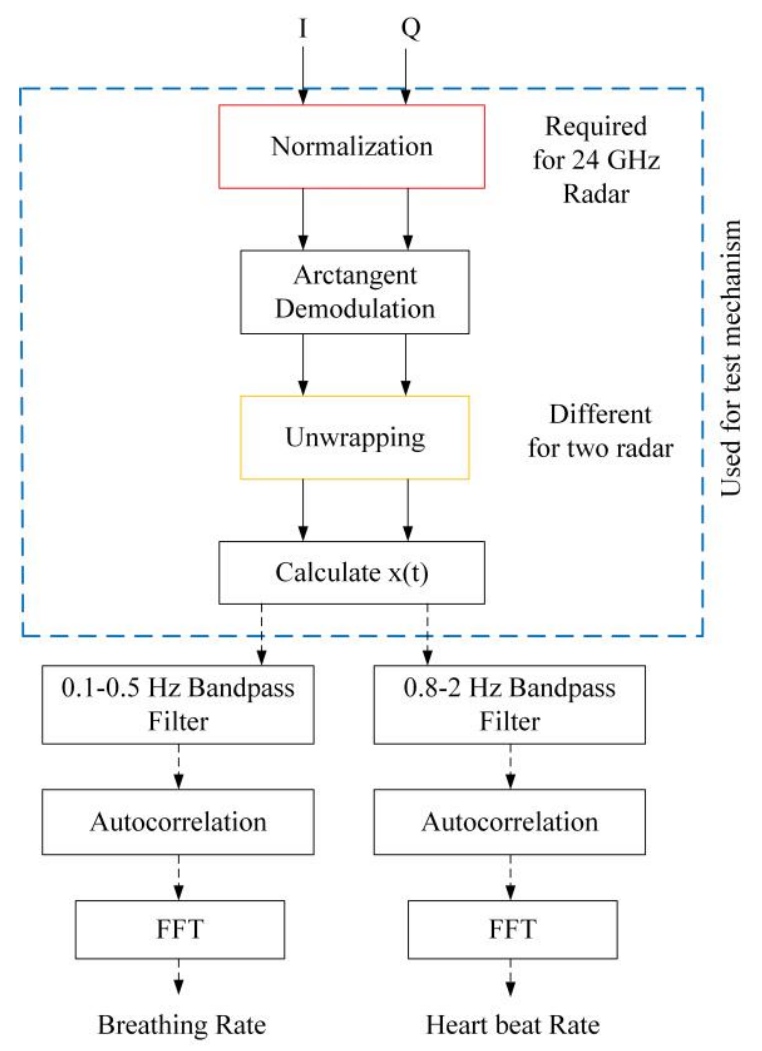

Fig. 7. Flow chart of the algorithms.

Although normalization is required for quadrature baseband signals of the $24 \mathrm{GHz}$ radar, it is not required for the $2.4 \mathrm{GHz}$ radar algorithm. Normalization is given in (7)

$$
\left\{\begin{array}{l}
I_{\text {norm }}=\frac{I-I_{\min }}{I_{\max }+I_{\min }}, \\
Q_{\text {norm }}=\frac{Q-Q_{\min }}{Q_{\max }+Q_{\min }} .
\end{array}\right.
$$

In (7), $I_{\text {norm }}$ and $Q_{\text {norm }}$ show normalized radar outputs, whereas $I$ and $Q$ instantaneous outputs, $I_{\min }, Q_{\min }$ and $I_{\text {max }}$ $Q_{\max }$ demonstrate the minimum and maximum radar outputs obtained during 60 seconds measurements, respectively. Both radars use arctangent demodulation to extract the phase information from baseband signals. The arctangent function takes values in the range of $(-\pi / 2, \pi / 2)$. If $x(t)$ is larger compared to $\lambda / 2$, demodulation can exceed these values, so that phase discontinuities occur. A movement of length $\lambda / 2$ corresponds to a change of $2 \pi$ in the phase of signal. The wavelengths for the $24 \mathrm{GHz}$ and $2.4 \mathrm{GHz}$ radars are $1.25 \mathrm{~cm}$ and $12.5 \mathrm{~cm}$, respectively. Therefore, movements above $6.25 \mathrm{~mm}$ for $24 \mathrm{GHz}$ radar and $62.5 \mathrm{~mm}$ for $2.4 \mathrm{GHz}$ radar cause the phase information exceeding $2 \pi$. In addition, DC components, phase and amplitude mismatches in the channels cause discontinuity in the obtained signal's phase. To prevent this, unwrapping is applied to the phase. For the $2.4 \mathrm{GHz}$ radar, $\pm 2 \pi$ is added to the phase if the discontinuity is equal to or greater than $\pi$. For the $24 \mathrm{GHz}$ radar, firstly, the number of complete turns of the phase change corresponding to the targeted movement on the unit circle are calculated. $\pm \pi$ offset values are added if the phase values are greater than $0.99 \pi$. The expression in (8) is used to obtain the motion signal $x(t)$ from the unwrapped phase information

$$
x(t)=\frac{\phi_{\text {unwrapped }}(t) \lambda}{4 \pi} .
$$

The operations up to this part of the algorithm are sufficient to obtain chest wall movement. This part is used for the test mechanism. In order to obtain vital signs, the motion signal is filtered by two separate high order band pass filters. The frequency bands of the filters used to obtain the respiration and heartbeat rate are from $0.1 \mathrm{~Hz}$ to $0.5 \mathrm{~Hz}$ and from $0.8 \mathrm{~Hz}$ to $2 \mathrm{~Hz}$, respectively. Frequency information of respiration and heartbeat signals is obtained by applying Fast Fourier Transform (FFT) to the filtered signals. Autocorrelation is performed to increase periodicity before FFT. Finally, the rate of respiration and heartbeat per minute is determined.

\section{Measurement Results}

Initially, a target test mechanism, which performs a small vibration at a fixed distance, is set up. The mechanism moves a 20x30 copper plate by using a step motor with driver. It is used to simulate chest wall motion by adjusting the movement to $8.5 \mathrm{~mm}$. Vibration frequency is $0.25 \mathrm{~Hz}$. The target test mechanism is displayed in Fig. 8.

For measurements performed with Radar 1, the mechanism is located at a distance of $1 \mathrm{~m}$ from the radar. The measurement time is set to 60 seconds. As a result of the measurements, movement is determined as $8.28 \mathrm{~mm}$ and the vibration frequency is $0.244 \mathrm{~Hz}$. The displacement and vibration frequency from measurement results for Radar 1 are demonstrated in Fig. 9.

Similar measurements are made with the Radar 2. The distance between the radar antenna and the test mechanism is $1 \mathrm{~m}$. Radar 2 measures the displacement $9.03 \mathrm{~mm}$ while finding the same vibration frequency as Radar 1 . Figure 10 
depicts the results for Radar 2.

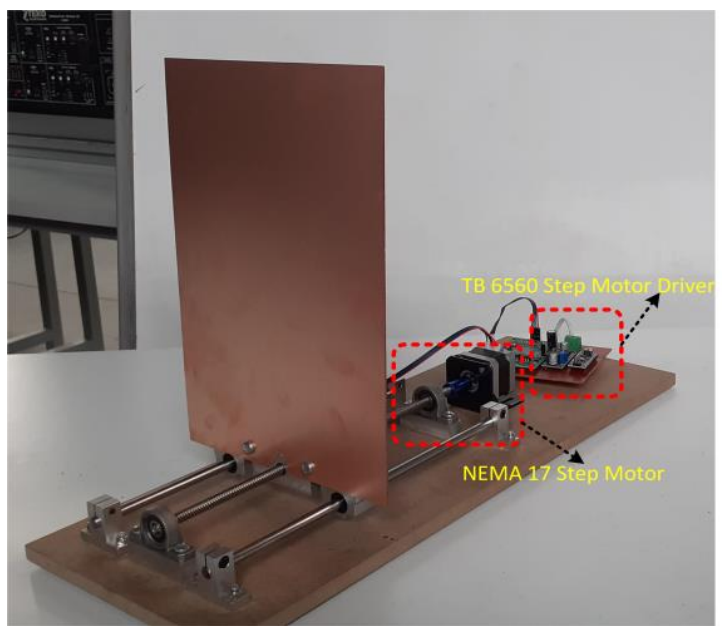

Fig. 8. The target test mechanism.

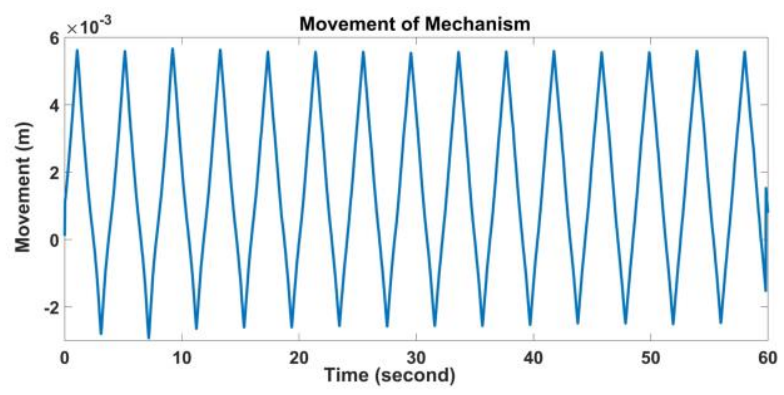

(a)

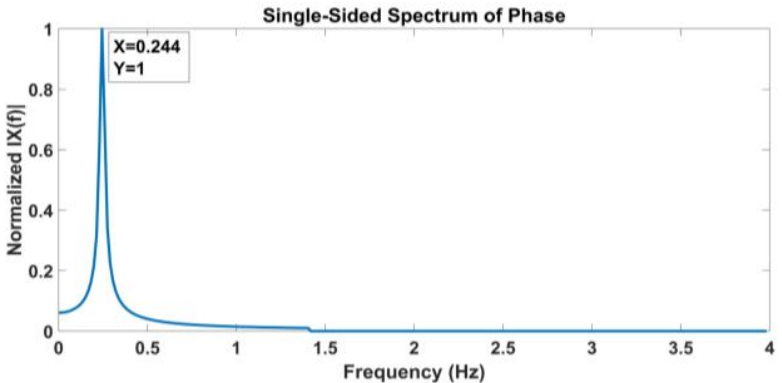

(b)

Fig. 9. (a) Movement over time; (b) Vibration frequency for Radar 1.

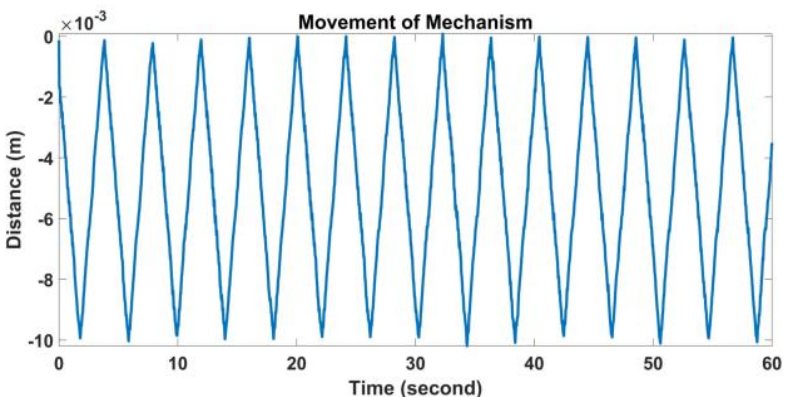

(a)

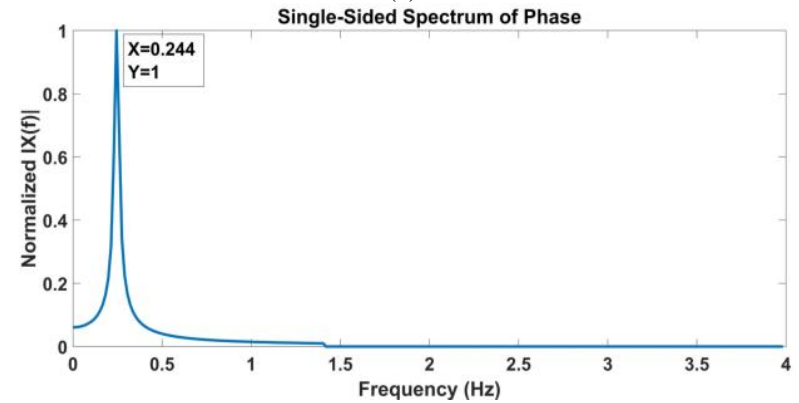

(b)

Fig. 10. (a) Movement over time; (b) Vibration frequency for Radar 2.
Relative error (RE) and percentage error (PE) of the results attained from both radars with the target test mechanism are tabulated in Table III.

TABLE III. TARGET TEST MECHANISM MEASUREMENT ERRORS

\begin{tabular}{|c|c|c|}
\hline Error (for Movement) & Radar 1 (24 GHz) & Radar 2 (2.4 GHz) \\
\hline RE & 0.02 & 0.06 \\
\hline PE & $2.58 \%$ & $6.23 \%$ \\
\hline $\begin{array}{c}\text { Error (for Vibration } \\
\text { frequency) }\end{array}$ & Radar 1 (24 GHz) & Radar 2 (2.4 GHz) \\
\hline RE & 0.024 & 0.024 \\
\hline PE & $2.4 \%$ & $2.4 \%$ \\
\hline
\end{tabular}

Measurements are then performed on a healthy human subject for both radars. For both radars, the subject is seated $1.5 \mathrm{~m}$ away from the radar antenna. Measurements are fulfilled for 60 seconds. Veron VRN 501 finger pulse oximeter is used as respiration and heartbeat references. Another reference for respiration measurements is the counting of respiration during the measurement.

Measurement results for Radar 1 are given in Fig. 11. The upper and middle figures show I and Q radar signals, while the lower figure displays the movement carried out by the subject. Similar results are presented in Fig. 12 for Radar 2.

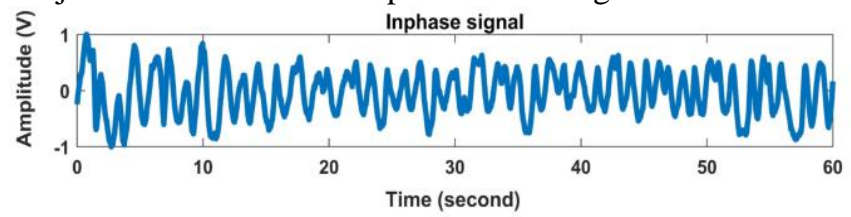

(a)

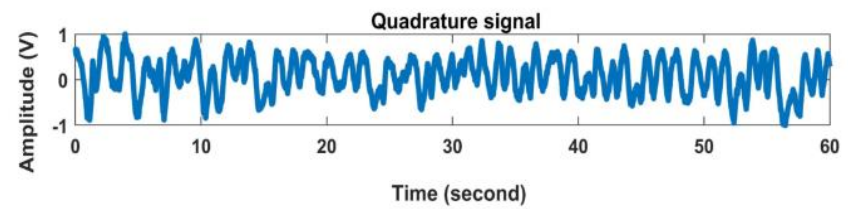

(b)

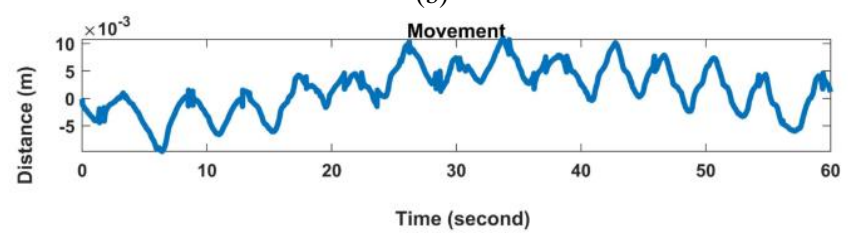

(c)

Fig. 11. Measurement results for Radar 1: (a) I signal; (b) Q signal; (c) Movement over time

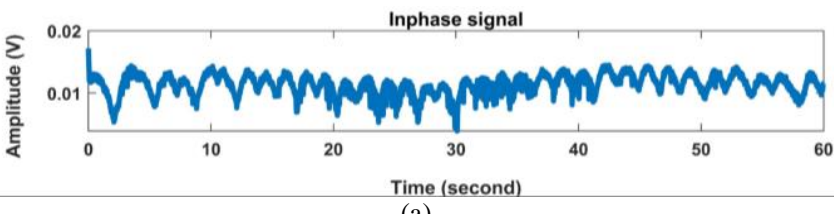

(a)

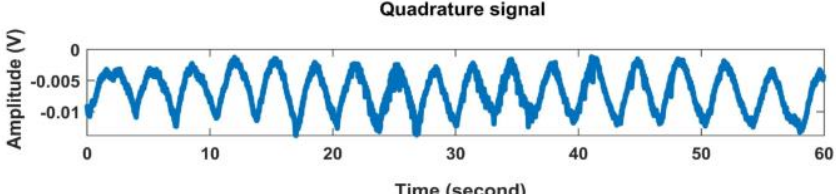

(b)

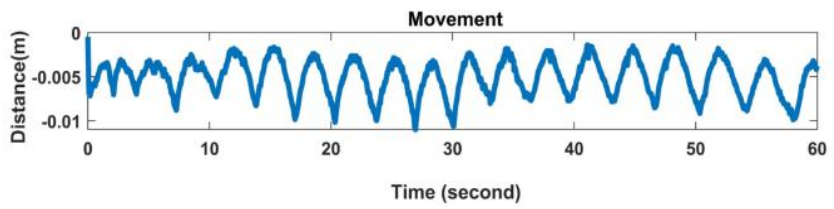

(c)

Fig. 12. Measurement results for Radar 2: (a) I signal; (b) Q signal; (c) Movement over time 
The movement frequency of the subject measured by Radar 1 and Radar 2 is different in Fig. 11 and Fig. 12 because the measurement occurs at different times with the same subject. The subject's posture, physiology, and psychology affect this situation. Respiration and heartbeat signals for both radars are presented in Fig. 13 and Fig. 14, respectively. It is understood from the figures that the results can also be reached with peak detection algorithms. However, FFT is preferred in this study.

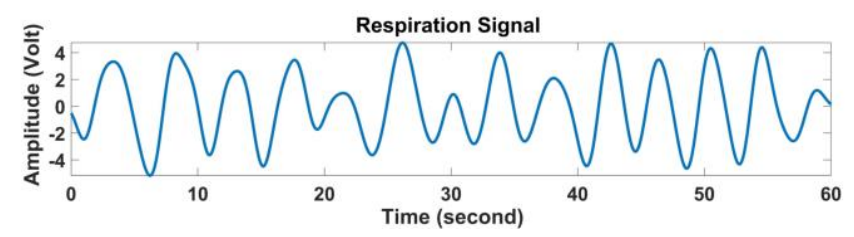

(a)

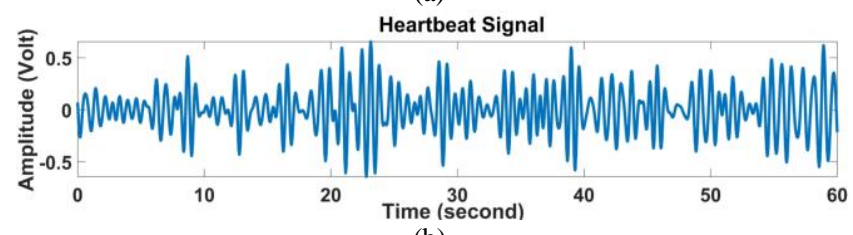

(b)

Fig. 13. (a) Respiration signal; (b) Heartbeat signal for Radar 1.

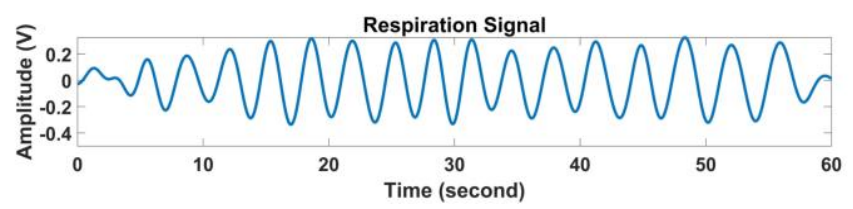

(a)

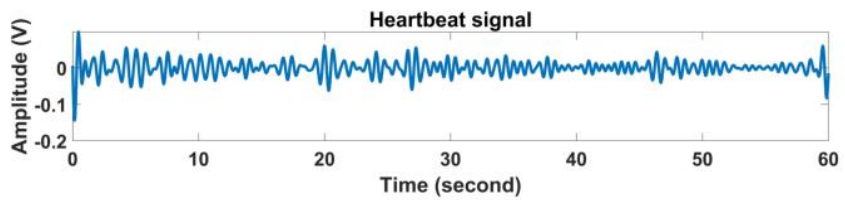

(b)

Fig. 14. (a) Respiration signal; (b) Heartbeat signal for Radar 2.

The respiration frequency is calculated by taking the FFT of the respiration signal during the measurement period. As a result of the measurement for Radar 1, the calculated respiration frequency is $0.229 \mathrm{~Hz}$. This value is ascertained as $0.29 \mathrm{~Hz}$ in the measurement of Radar 2. Fig. 15 and Fig. 16 illustrate these results.

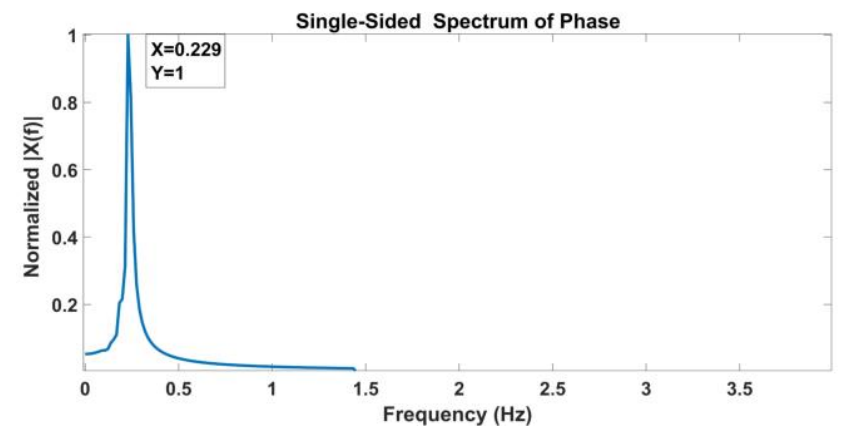

Fig. 15. Frequency spectrum of the measured respiration signal for Radar 1 .

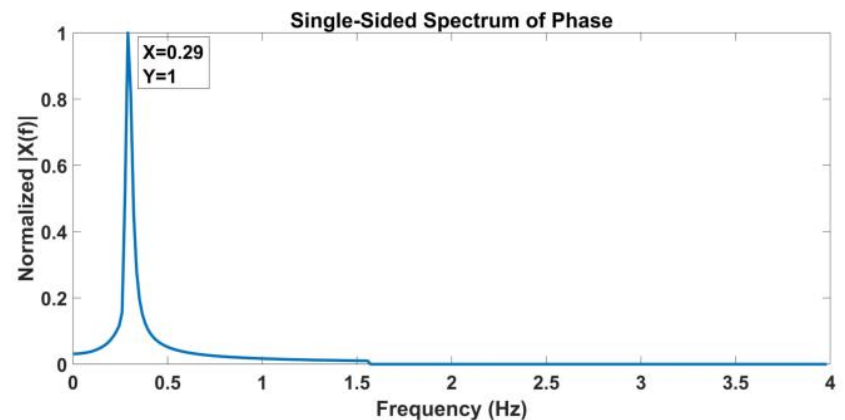

Fig. 16. Frequency spectrum of the measured respiration signal for Radar 2 .

The difference in measurement between respiration and heart rate measured by Radar 1 and Radar 2 from Fig. 13 to Fig. 16 is a result of measurements of the same subject at different times, as previously stated. Frequency of heart rate is calculated in the same way as obtaining the respiration frequency. As a result of these measurements for Radar 1 and Radar 2, these values are found as $1.251 \mathrm{~Hz}$ and $1.541 \mathrm{~Hz}$, respectively. Fig. 17 and Fig. 18 reveal the frequency of heartbeat rate.

For two radars, the results acquired from the measurements performed with human subject and the comparisons with reference values and error rates are given in Table IV.

TABLE IV. HUMAN SUBJECT MEASUREMENT COMPARISONS.

\begin{tabular}{|c|c|c|c|c|c|c|}
\hline \multicolumn{2}{|c|}{$\begin{array}{c}\text { Radar 1 } \\
(24 \mathrm{GHz}) \\
\text { For five measurements }\end{array}$} & \multirow[t]{2}{*}{$\begin{array}{c}\text { Ref. } \\
\text { (Veron) } \\
\text { (Resp.) }\end{array}$} & \multirow[t]{2}{*}{$\begin{array}{l}\text { Ref. } \\
\text { (Counted) } \\
\text { (Resp.) }\end{array}$} & \multirow[t]{2}{*}{$\begin{array}{c}\text { Ref. } \\
\text { (Veron) } \\
\text { (Heart) }\end{array}$} & \multirow[t]{2}{*}{$\begin{array}{c}\text { PE } \\
\text { (Resp.) }\end{array}$} & \multirow[t]{2}{*}{$\begin{array}{c}\text { PE } \\
\text { (Heart) }\end{array}$} \\
\hline Resp. & Heart & & & & & \\
\hline 13.74 & 75.06 & 14 & 14 & 80 & $\% 1.85$ & $\% 6.17$ \\
\hline 14.4 & 87 & 15 & 14 & 81 & $\% 4$ & $\% 7.40$ \\
\hline 9.54 & 63.6 & 10 & 9 & 70 & $\% 4.6$ & $\% 9.14$ \\
\hline 19.2 & 88.8 & 20 & 19 & 95 & $\% 4$ & $\% 6.52$ \\
\hline 24.3 & 96.4 & 25 & 25 & 103 & $\% 2.8$ & $\% 6.4$ \\
\hline \multicolumn{2}{|c|}{$\begin{array}{c}\text { Radar } 2 \\
(2.4 \text { GHz }) \\
\text { For five measurements }\end{array}$} & $\begin{array}{c}\text { Ref. } \\
\text { (Veron) } \\
\text { (Resp.) }\end{array}$ & $\begin{array}{c}\text { Ref. } \\
\text { (Counted) } \\
\text { (Resp.) }\end{array}$ & $\begin{array}{c}\text { Ref. } \\
\text { (Veron) } \\
\text { (Heart) }\end{array}$ & $\begin{array}{c}\text { PE } \\
\text { (Resp.) }\end{array}$ & $\begin{array}{c}\text { PE } \\
\text { (Heart) }\end{array}$ \\
\hline Resp. & Heart & & & & & \\
\hline 17.4 & 92.46 & 17 & 18 & 84 & $\% 2.35$ & $\% 9.15$ \\
\hline 14.76 & 81.62 & 14 & 14 & 90 & $\% 5.43$ & $\% 9.31$ \\
\hline 11.34 & 75.24 & 11 & 12 & 82 & $\% 3.09$ & $\% 8.24$ \\
\hline 16.38 & 82.8 & 16 & 16 & 91 & $\% 2.37$ & $\% 9.01$ \\
\hline 20.37 & 88.14 & 21 & 20 & 98 & $\% 3$ & $\% 9.13$ \\
\hline
\end{tabular}




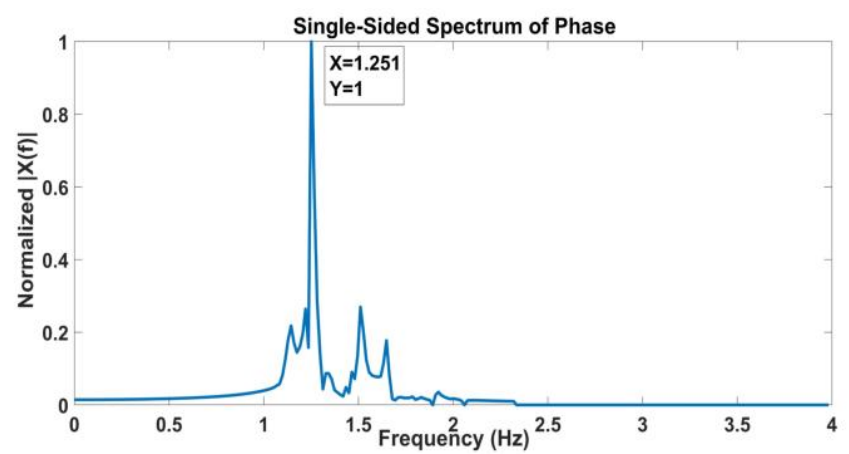

Fig. 17. Frequency spectrum of the measured heartbeat signal for Radar 1.

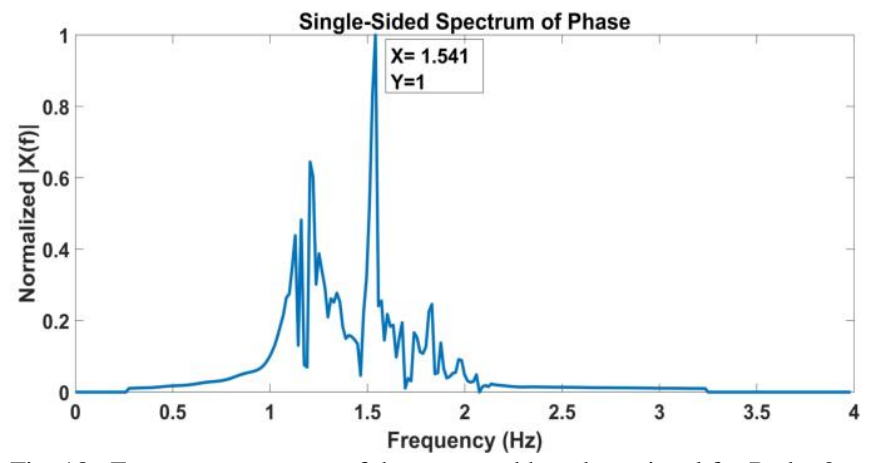

Fig. 18. Frequency spectrum of the measured heartbeat signal for Radar 2.

\section{CONCLUSIONS}

In this paper, the basic structure and operating principle of continuous wave Doppler radar are explained and how Doppler radar provides non-contact motion detection is submitted. A low-cost radar is manufactured on a printed circuit board (PCB) with the necessary circuit elements and commercial - off-the-shelf radar. In addition, another radar structure is implemented by combining discrete components. First, a small movement is detected with the help of a target test mechanism. For motion detection with radars operating at $24 \mathrm{GHz}$ and $2.4 \mathrm{GHz}$, PEs are $2.58 \%$ and $6.23 \%$, respectively. $\mathrm{PE}$ for the vibration frequency of the mechanism is $2.4 \%$ for both radars. Experiments are then carried out with a human subject to acquire non-contact vital signs. For the $24 \mathrm{GHz}$ radar, respiration rate and heartbeat rate determined with PEs are $1.85 \%$ and $6.17 \%$, respectively. These error values for the other radar are $2.35 \%$ and $8.24 \%$. From the measurement results, it can be seen that the resolution of the high frequency radar is better than that of the low frequency radar. Also, the designed radar structures and the proposed algorithms are usable for vital sign detection with a high accuracy. Measurement results indicate that radars may find a wider use in the field of medicine in the future.

\section{CONFLICTS OF INTEREST}

The authors declare that they have no conflicts of interest.

\section{REFERENCES}

[1] World Population Prospects 2019: Highlights, United Nations, Department of Economic and Social Affairs, Population Division, New York, NY, USA, 2019.
[2] M. I. Skolnik, Radar Handbook, 3rd ed. New York, McGraw-Hill Book Co, 2008, ch. 1.

[3] M. Amin, Radar for Indoor Monitoring: Detection, Classification, and Assessment. Florida, CRC Press, 2017, ch. 1. DOI: 10.1201/9781315155340.

[4] J. Kuutti, M. Paukkunen, M. Aalto, P. Eskelinen, and R. E. Sepponen, "Evaluation of a Doppler radar sensor system for vital signs detection and activity monitoring in a radio-frequency shielded room", Measurement, vol. 68, pp. 135-142, 2015. DOI: 10.1016/j.measurement.2015.02.048.

[5] F. Khan and S. Cho, "A detailed algorithm for vital sign monitoring of a stationary/non-stationary human through IR-UWB radar", Sensors, vol. 17, no. 2, p. 290, 2017. DOI: 10.3390/s17020290.

[6] M. Alizadeh, G. Shaker, J. C. M. De Almeida, P. P. Morita, and S Safavi-Naeini, "Remote monitoring of human vital signs using mmwave FMCW radar", IEEE Access, vol. 7, pp. 54958-54968, 2019. DOI: 10.1109/ACCESS.2019.2912956.

[7] S. Nahar, T. Phan, F. Quaiyum, L. Ren, A. E. Fathy, and O. Kilic, "An electromagnetic model of human vital signs detection and its experimental validation", IEEE Journal on Emerging and Selected Topics in Circuits and Systems, vol. 8, no. 2, pp. 338-349, 2018. DOI: 10.1109/JETCAS.2018.2811339.

[8] R. Vasireddy, C. Roth, J. Mathis, J. Goette, M. Jacomet, and A. Vogt, "K-band Doppler radar for contact-less overnight sleep marker assessment: A pilot validation study", Journal of clinical monitoring and computing, vol. 32, no. 4, pp. 729-740, 2018. DOI: 10.1007/s10877-017-0060-9.

[9] F. Qi, C. Li, S. Wang, H. Zhang, J. Wang, and G. Lu, "Contact-free detection of obstructive sleep apnea based on wavelet information entropy spectrum using bio-radar", Entropy, vol. 18, no. 8, p. 306, 2016. DOI: $10.3390 / \mathrm{e} 18080306$.

[10] C. Li and J. Lin, Microwave noncontact motion sensing and analysis. New Jersey, John Wiley \& Sons, 2013, ch.5. DOI: $10.1002 / 9781118742556$.

[11] L. Anishchenko, A. Zhuravlev, and M. Chizh, "Fall detection using multiple bioradars and convolutional neural networks", Sensors, vol. 19, no. 24, p. 5569, 2019. DOI: 10.3390/s19245569.

[12] H. Wang, D. Zhang, Y. Wang, J. Ma, Y. Wang, and S. Li, "RT-fall: A real-time and contactless fall detection system with commodity WiFi devices", IEEE Trans. Mob. Comput., vol. 16, pp. 511-526, 2017. DOI: 10.1109/TMC.2016.2557795.

[13] K. M. Chen, Y. Huang, J. Zhang, and A. Norman, "Microwave lifedetection systems for searching human subjects under earthquake rubble or behind barrier", IEEE Transactions on Biomedical Engineering, vol. 47, no. 1, pp. 105-114, 2000. DOI: $10.1109 / 10.817625$

[14] A. Kılıç, İ. Babaoğlu, A. Babalık, and A. Arslan, "Through-wall radar classification of human posture using convolutional neural networks", International Journal of Antennas and Propagation, vol. 2019, 2019. DOI: $10.1155 / 2019 / 7541814$.

[15] L. Liu, Z. Liu, and B. E. Barrowes, "Through-wall bio-radiolocation with UWB impulse radar: Observation, simulation and signal extraction", IEEE Journal of Selected Topics in Applied Earth Observations and Remote Sensing, vol. 4, no. 4, pp. 791-798, 2011. DOI: 10.1109/JSTARS.2011.2157461.

[16] Y. Jin, B. Kim, S. Kim, and J. Lee, "Design and implementation of FMCW surveillance radar based on dual chirps", Elektronika ir Elektrotechnika, vol. 24, no. 6, pp. 60-66, 2018. DOI: 10.5755/j01.eie.24.6.22292.

[17] L. Anishchenko, G. Gennarelli, A. Tataraidze, E. Gaysina, F. Soldovieri, and S. Ivashov, "Evaluation of rodents' respiratory activity using a bioradar", IET Radar, Sonar \& Navigation, vol. 9, no. 9, pp. 1296-1302, 2015. DOI: 10.1049/iet-rsn.2014.0553.

[18] K. Evteeva, A. Turetskaya, and L. Anishchenko, "Sensing of human breathing and heart beating at different orientation angles by $\mathrm{CW}$ Doppler radar", in Proc. of 2019 Ural Symposium on Biomedical Engineering, Radioelectronics and Information Technology (USBEREIT), Yekaterinburg, Russia, 2019. DOI: 10.1109/USBEREIT.2019.8736585.

[19] B. Park, O. Boric-Lubecke, and V. M. Lubecke, "Arctangent demodulation with DC offset compensation in quadrature Doppler radar receiver systems", IEEE Transactions on Microwave Theory and Techniques, vol. 55, no. 5, pp. 1073-1079, 2007. DOI: 10.1109/TMTT.2007.895653.

[20] K-LC6 Radar Transceiver. [Online]. Available: https://www.rfbeam.ch/product?id=12 